\begin{tabular}{|c|c|}
\hline \multicolumn{2}{|c|}{ Access this article online } \\
\hline \\
\hline
\end{tabular}

\title{
Comparative analysis of corneal thickness parameters using Scheimpflug imaging system and HD-OCT in keratoconic eyes
}

\author{
Magdalena Maleszka-Kurpiel ${ }^{1,2}$, Andrzej Michalski' ${ }^{2}$, Marta Robak ${ }^{1}$, Wojciech Warchoł ${ }^{2}$ \\ 'Optegra Eye Health Care Clinic in Poznan, Poland \\ ${ }^{2}$ Department of Optometry, Chair of Ophthalmology and Optometry, Poznan University of Medical Sciences, Poland
}

\begin{abstract}
Introduction: The Scheimpflug camera and optical coherence tomography (OCT) are important tools in diagnosis and followup of keratoconus. In our study we evaluated and compared the automatic measurement results provided in keratoconic eyes with the Scheimpflug camera and HD-OCT. Central corneal thickness (CCT), thinnest corneal thickness (TCT) and Y-coordinate of the thinnest point (Y-TCT) were analyzed.

Material and methods: 66 eyes of 33 patients diagnosed with keratoconus were included in the study. Each patient underwent examination with a Scheimpflug camera (WaveLight Oculyzer II) and HD-OCT with cornea lens attachment (Zeiss Cirrus 5000) on the same visit.

Results: We did not find a statistically significant difference in CCT value derived with the two imaging systems: $478 \pm 47 \mu \mathrm{m}$ (Oculyzer), $477 \pm 47 \mu \mathrm{m}$ (HD-OCT). TCT measured with the HD-OCT $(455 \pm 45 \mu \mathrm{m})$ was thinner than measured with the Oculyzer $(466 \pm 46 \mu \mathrm{m})$. Y-TCT and $\Delta$ CCT-TCT were not well correlated
\end{abstract}

between imaging systems and characterized by greater variance for HD-OCT. No statistically significant difference in the number of eyes counted in the study group was obtained using the cut-off points values recommended by 22 . Ambrósio et al. for the diagnosis of keratoconus for CCT, TCT, Y-TCT, and $\Delta$ CCT-TCT.

Conclusions: We do not recommend using the Scheimpflug camera and HD-OCT interchangeably in the diagnosis and monitoring of patients with keratoconus. Although the corneal thickness measurement results are highly correlated, these cannot be used interchangeably in clinical practice except for CCT. Vertical TCT decentration and $\triangle$ CCT-TCT show a weak correlation. Analysis of the number of eyes using the cut-off point values published for data derived with the Scheimpflug camera suggests that these could also be used for data derived with HD-OCT.

KEY WORDS: pachymetry, keratoconus, scheimpflug camera, thinnest corneal thickness vertical decentration, HD-OCT with cornea lens attachment.

\section{INTRODUCTION}

Keratoconus $(\mathrm{KC})$ is defined as a non-inflammatory, bilateral ectatic disease causing progressive corneal thinning and steepening [1].

Keratoconus affect both genders, and data about gender predilection are not consistent. Li et al. found no difference between genders, Wagner et al. found $\mathrm{KC}$ more frequently in males $[2,3]$.

Keratoconus has a multifactorial etiological background, in which genetic and environmental factors are involved [4]. The disease begins in adolescence and progresses through the third and/or fourth decades of life. It has to be noted that progression differs among patients. $\mathrm{KC}$ has considerable implications in the public health area [1]. As reported by Srujana et al., visual impairment due to KC significantly affects quality of life [5].

Accurate corneal imaging is necessary to diagnose keratoconus, especially in its initial stages, when visual acuity is unaffected. The good repeatability and reproducibility of the values measured are of utmost importance, as they enable an accurate follow-up to detect keratoconus progression and the effects of corneal cross-linking (CXL). Corneal thickness is an important marker for both detection of $\mathrm{KC}$ and grading of the disease [6-8], because corneal thinning is a key pathologic feature of keratoconus [9].

The exact thickness and location of the thinnest point of the cornea (TCT) are clinically crucial in planning CXL surgery and then directly before irradiation. It is proven that 
UVA radiation reaches $300 \mu \mathrm{m}$ - in corneas thinner than $400 \mu \mathrm{m}$ it can damage the endothelium [10-12].

Measurement of corneal thickness has also become an important factor in planning refractive surgery and evaluation of its results, estimating an individual risk factor for glaucoma, monitoring corneal edema and predicting graft survival after penetrating keratoplasty [13-17].

Ultrasound modality is still often used to assess pachymetry. It is inexpensive, but burdened with several disadvantages caused mainly by probe-cornea contact [18].

Currently different noncontact imaging modalities are used for the measurement of pachymetry, including devices with a Scheimpflug camera, and instruments based on optical coherence tomography (OCT).

In the Scheimpflug system the rotating camera creates an image by intersecting the eye plane with a slit beam. After collecting a set of slit images in a short time software is used to render a 3D image. This system determines net corneal power, elevation maps, pachymetry map, curvature (front and back) maps, anterior chamber depth, and corneal wavefront [19].

Optical coherence tomography is a noncontact technology that produces high-resolution cross-sectional images enabling the precise visualization of anterior segment structure. AS-OCT imaging was first introduced in 1994 [20]. Nowadays spectral domain OCT systems (SD-OCT) with corneal and anterior chamber lenses are able to measure pachymetry, epithelial thickness iridocorneal angle, and detailed anterior chamber dimensions [21].

Advances in the diagnostic technologies resulted in the development of new methods of the measurement of pachymetry and other corneal parameters. Since CCT measurements might be performed using different types of equipment, knowledge about correlation of the results obtained with distinctive devices is crucial for the follow-up of the patients.

\section{AIM OF THE STUDY}

Comparison of CCT, TCT, Y-coordinate of TCT measured with a Scheimpflug camera and HD-OCT with cornea lens in patients with KC.

\section{MATERIAL AND METHODS}

In the study, we included 66 eyes of 33 Caucasian patients (27 males and 6 females) diagnosed with keratoconus.

The mean age in the study group was 27 years. Each patient underwent examination with the Scheimpflug imaging system and HD-OCT with a cornea lens (pachymetry scan) on the same visit. We included only good quality results in the study. Both tests were performed one after another and prior to administration of any eyedrops. We excluded eyes with corneal scarring or previous surgery (including cross-linking). Patients did not use contact lenses for 1 month preceding examination.

Scheimpflug imaging was obtained by WaveLight Oculyzer ${ }^{\mathrm{II}}$ (Alcon, Texas, United States), and results of measurements of corneal thickness at the apex (CCT), corneal thickness at thinnest point (TCT), and vertical coordinate of TCT (Y-TCT), were taken for further analysis. Images were performed automatically when the camera was centered on the corneal apex. Only scans of proper quality (quality specification 'OK') were included for further analysis.

Central corneal thickness, TCT and Y-TCT were also obtained by OCT Cirrus HD OCT 5000 (Carl Zeiss Meditec, Jena, Germany). Pachymetry scan requires the cornea external lens. Images were performed when the scan line was placed on the corneal apex. The pachymetry was analyzed automatically in seventeen sectors of the cornea.

Y-coordinate of TCT was given in cartesian coordinates with the value 0 at the corneal apex and negative values corresponding to location below the corneal apex along the vertical axis.

In our study, we evaluated only data which are obtained using both devices.

The Shapiro-Wilk test was used for the evaluation of the distribution of continuous variables. Normally distributed data are shown as mean \pm standard deviation, while non-normally distributed variables are presented as a median and range (minimum-maximum). Paired Student's $t$-test was used for analysis of normally distributed outcomes from both devices ( $p$ from 0.189 to 0.979 , Shapiro-Wilk test) and Wilcoxon test for non-normally distributed $\Delta$ CCT-TCT (calculated parameter). The F-test was applied for comparison of variability of acquired data. Dichotomous data were compared using Fisher's exact test. All statistical analyses were performed using Statistica version 13 (TIBCO Software Inc., CA, United States).

The study was approved by the ethics committee of Poznan University of Medical Sciences. All subjects received an explanation about the study and provided written consent.

\section{RESULTS}

Table I presents the results of pachymetry measured by the two different imaging systems. Differences are statistically significant except for CCT. $\Delta$ CCT-TCT is on average $8.5 \mu \mathrm{m}$ greater for Zeiss.

We found a strong positive correlation for CCT $(R=0.927$, $p<0.001$; Figure 1$)$ and TCT $(R=0.927, p<0.001$; Figure 2$)$ measured with the two systems. The correlations for Y-TCT and $\triangle$ CCT-TCT were also positive but weaker $(R=0.540$, $p<0.001$, Figure 3; $R=0.524, p<0.001$, Figure 4; respectively). Data for CCT and TCT were homogeneous for both devices $(F<0.001, p=0.990 ; F=0.001, p=0.973$, respectively). Data for Y-TCT and $\triangle$ CCT-TCT did not meet the requirement of homogeneity, with variability greater for Zeiss $(F=12.799$, $p<0.001 ; F=21.001, p<0.001$, respectively). We also count-

Table I. CCT, TCT, Y-TCT $[\mu \mathrm{m}]$ presented as mean value \pm SD; $\Delta$ CCT-TCT $[\mu \mathrm{m}]$ presented as median and range; $p$ : $t$-test for dependent means, ${ }^{*}$ Wilcoxon test

\begin{tabular}{|l|c|c|c|}
\hline Parameter & Oculyzer II & Zeiss Cirrus 5000 & $p$ \\
\hline CCT $[\mu \mathrm{m}]$ & $478 \pm 47$ & $477 \pm 47$ & 0.586 \\
\hline TCT $[\mu \mathrm{m}]$ & $466 \pm 46$ & $455 \pm 45$ & $<0.001$ \\
\hline Y-TCT $[\mu \mathrm{m}]$ & $-627 \pm 281$ & $-881 \pm 470$ & $<0.001$ \\
\hline$\Delta$ CСТ-TCT $[\mu \mathrm{m}]$ & $12.5[2-34]$ & $21[1-72]$ & $<0.001^{*}$ \\
\hline
\end{tabular}




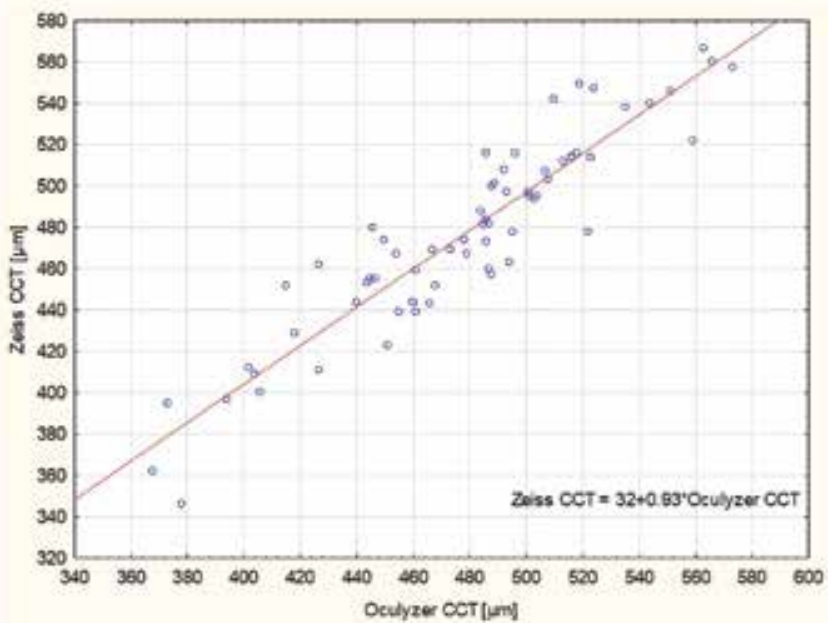

Figure 1. CCT (Zeiss Cirrus 5000) as a function of CCT (Oculyzer II)

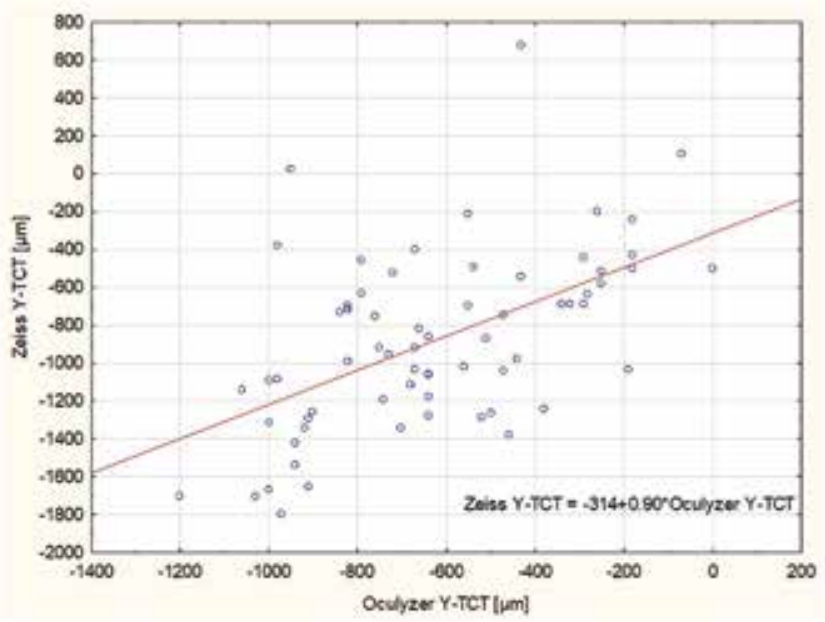

Figure 3. Y-TCT (Zeiss Cirrus 5000) as a function of Y-TCT (Oculyzer II)

ed the number of eyes using cut-off point values reported by Ambrósio et al. [22]. Number of eyes counted with parameters derived with the Zeiss device was greater for all criteria except for CCT, but there was no statistical significance in Fisher's exact test for any of them (Table II).

\section{DISCUSSION}

Establishing repeatability and reproducibility of the acquired examination results is crucial for confident use of these in clinical practice. In our study we checked the reproducibility of measurements of selected corneal parameters acquired by two different imaging devices, and considered whether it is possible to use these results interchangeably. Both the HD-OCT device and Scheimpflug imaging system are noncontact devices and are well tolerated by patients. Knowledge of interdevice reproducibility is especially important in patients who are followed up in different ophthalmological offices.

Central corneal thickness, TCT and vertical distance from the thinnest point to the corneal vertex are among the most important parameters in diagnosis and monitoring of pro-

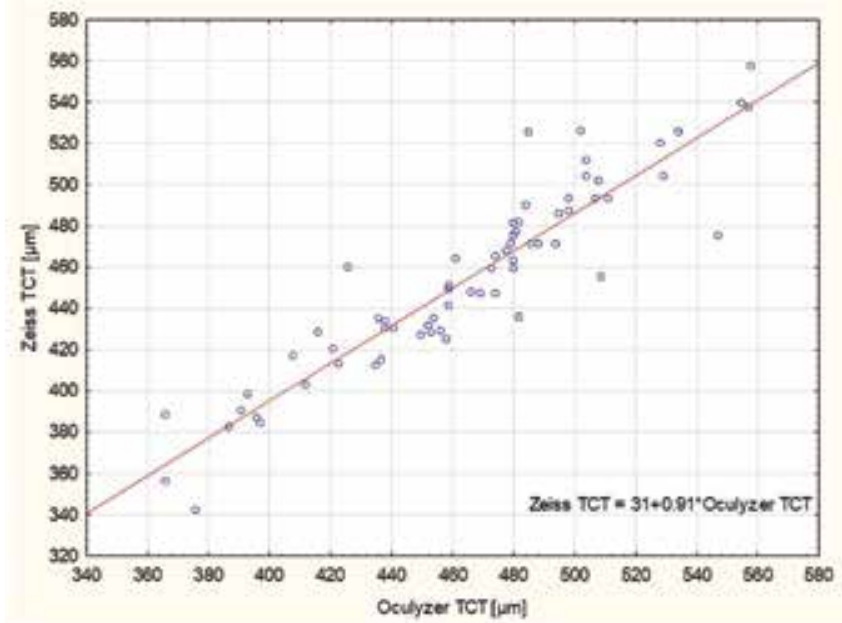

Figure 2. TCT (Zeiss Cirrus 5000) as a function of TCT (Oculyzer II)

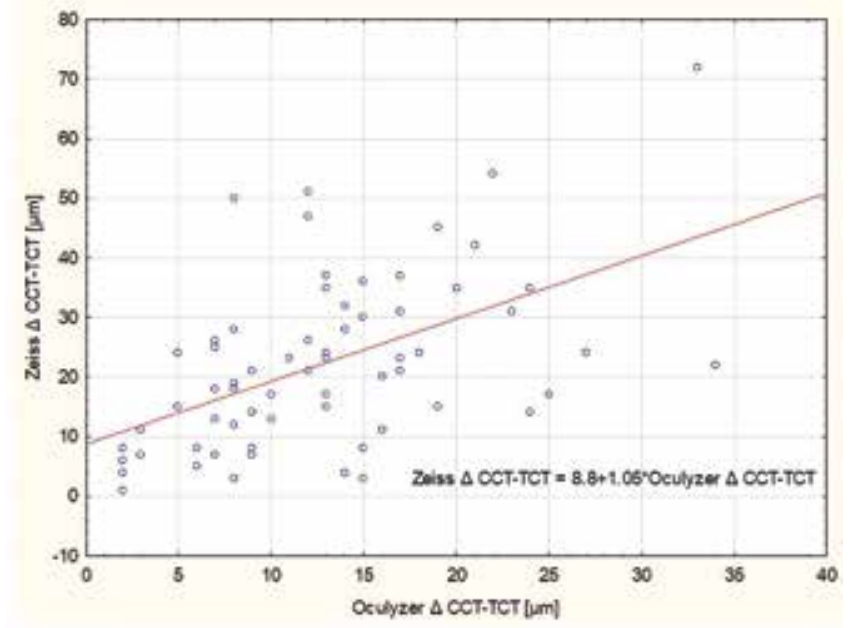

Figure 4. $\Delta$ CCT-TCT (Zeiss Cirrus 5000) as a function of $\Delta$ CCT-TCT (0culyzer II)

Table II. Number of eyes counted for cut-off points as per Ambrósio; $p$ : Fisher's exact test

\begin{tabular}{|l|c|c|c|}
\hline Number of eyes with & Oculyzer II & Zeiss Cirrus 5000 & $\boldsymbol{p}$ \\
\hline $\mathrm{CCT}<529 \mu \mathrm{m}$ & 59 & 57 & 0.791 \\
\hline $\mathrm{TCT}<504 \mu \mathrm{m}$ & 53 & 56 & 0.647 \\
\hline $\mathrm{Y}<-460 \mu \mathrm{m}$ & 47 & 55 & 0.145 \\
\hline$\Delta \mathrm{CCT}-\mathrm{TCT}>8 \mu \mathrm{m}$ & 44 & 51 & 0.245 \\
\hline
\end{tabular}

gression in $\mathrm{KC}$, the clinical usefulness of which is well documented [22-25].

There are different measurement technologies used for cornea examination, which can provide discrepancies in results.

The rotating Scheimpflug camera is a gold standard in KC diagnostics. Numerous studies have proved the repeatability of measurements in normal and keratoconic eyes $[26,27]$. However, it has to be noted that different systems using the Scheimpflug camera generate results which are not interchangeable in keratoconic patients [28]. 
The usefulness of OCT technique has been confirmed in detection and monitoring of keratoconus progression [24, 29]. Some researchers suggest using OCT only as a tool supporting the Scheimpflug camera [30]. Wang et al. draw attention to the discrepancy in data obtained by two OCT devices [31].

Numerous studies confirm that the Scheimpflug imaging system tends to overestimate CCT in comparison to OCT in healthy eyes $[32,33]$. Baghdasaryan et al. found a $13.46 \mu \mathrm{m}$ difference in CCT measured in healthy eyes with Cirrus HD OCT with a cornea lens and with Pentacam HR. Pachymetry measured with the latter device was thicker [21]. Kiraly et al. obtained a similar discrepancy $(11.44 \mu \mathrm{m})$ between Pentacam HR and Cirrus HD OCT 400 in healthy eyes [33].

In our results the difference in CCT measured with OCT and the Scheimpflug imaging system is not statistically significant, and it is similar to results obtained by Prospero et al. [34]. The results of other research comparing pachymetry results obtained with OCT and the Scheimpflug system are disparate. Yazici et al. reported a mean difference of $14.5 \mu \mathrm{m}$ in CCT in KC patients captured by OCT Visante $(462.0 \mu \mathrm{m})$ and Pentacam $(476.5 \mu \mathrm{m})$ [35]. Nakagawa et al. also stated that the CCT obtained with OCT is thinner than with the Scheimpflug based imaging in keratoconic eyes [36]. Grewal et al. found a contrary result and stated that CCT in keratoconic eyes measured with the Scheimpflug imaging system is about $2 \mu \mathrm{m}$ thinner than measured with AS-OCT [18]. In the study of Kumar et al. it was also found that the CCT measured with OCT was overestimated $(7 \mu \mathrm{m})$ in comparison to the Scheimpflug imaging system [37].

Central corneal thickness as a single measurement has documented limitations for long-term follow-up and detecting pre-keratoconus $[38,39]$. Despite this, CCT is still a valuable parameter in identifying KC. Keratoconus may develop despite a very thick cornea. Berti et al. described 2 cases of keratoconus with pachymetry over $600 \mu \mathrm{m}$ [40].

TCT is a valuable diagnostic parameter in detecting primary ectatic disease. Several studies have validated the use of TCT in the identification of KC [41-44]. The thinnest corneal thickness (TCT) measurement has emerged as an efficient diagnostic parameter in cases where the classical topographic keratoconus index is not suitable [25]. In our study the TCT value was greater for Oculyzer II, which is similar to results published by Yazici et al. [35].

These differences may be a result of tear film disorders. Fujimoto et al. found difference in CCT and TCT measurements by Scheimpflug imaging and OCT in dry eye disease. The discrepancy was greater in severe dry eye disease $[45,46]$. We did not do any dry eye test in our group.

The exact location of the thinnest corneal point can have clinical consequences, namely in the detection of the early stages of keratoconus, which are characterized by changes in corneal topography and an inferior decentration of the thinnest corneal point [25]. Also Vinciguerra and Camesasca proved that asymmetric and eccentric corneal thinning is characteristic for keratoconus [47]. Ambrósio et al. evinced that in KC mean Y-TCT position measured using the rotating Scheimpflug camera is $-740 \pm 450 \mu \mathrm{m}$ (inferior decentration) in comparison to $-0.29 \pm 0.35 \mu \mathrm{m}$ in healthy eyes [22]. Zhu et al. reported Y-TCT as $-640 \pm 394 \mu \mathrm{m}$ in $\mathrm{KC}$, but without a significant difference to normal eyes $(-580 \pm 459 \mu \mathrm{m})[48]$.

Our results of vertical displacement of the thinnest cornea point measured by the Scheimpflug camera are similar to previous studies [49]. Li et al. measured the Y-coordinate of the thinnest point in $\mathrm{KC}$ using AS-OCT, and got a result of $805 \mu \mathrm{m}$ for inferior displacement [25]. This value is comparable with our result.

Rüfer et al. also mentioned that repetition accuracy for the measurements of location of the thinnest point was rather poor, based on a high standard deviation of $\mathrm{x}$ and $\mathrm{y}$ coordinates, and attributed this to minor fixation deviations of the subject's eyes [50].

In published studies the difference between the thinnest and central (or peripheral) corneal thickness was significantly greater in eyes with keratoconus than in normal eyes [51, 52]. Ambrósio et al. found a difference of $34.64 \pm 39.33 \mu \mathrm{m}$ between CCT and TCT in keratoconic eyes using rotating Scheimpflug camera topography [22].

In our study, for statistical analysis, we used evaluation cut-off point values published by Ambrósio et al. for detection of KC for the Scheimpflug camera [22]. We did not find a statistically significant difference between number of eyes meeting the criteria examined with two imaging modalities. Based on the above we suggest that these cut-off point values can be used for HDOCT systems with a cornea lens.

This study has some limitations. The examined group is relatively small and we examined only keratoconic eyes. Another limitation is the lack of evaluation of intra- and inter-observer reproducibility of the measurements. We also did not perform any dry eye test in our group.

\section{CONCLUSIONS}

In $\mathrm{KC}$ eyes we did not find a statistically significant difference in CCT value between the two imaging systems; this parameter can be used interchangeably. TCT measured with Zeiss was thinner. Y-TCT and $\Delta$ CCT-TCT are not well correlated between the two imaging systems and are characterized by greater variance for Zeiss.

We suggest that the cut-off point values calculated for the Scheimpflug imaging system can be used for HD-OCT with cornea lens attachment. Further work is required to find specific cut-off points in KC diagnosis for CCT, TCT, Y-TCT and $\triangle$ CCT-TCT for HD-OCT systems with a cornea lens.

When monitoring or diagnosing, discrepancies in results delivered with the two imaging modalities should be taken into account. We do not recommend using the Scheimpflug camera device and OCT device interchangeably. Although partially highly correlated, the measurements are not directly interchangeable in clinical practice.

\section{DISCLOSURE}

The authors declare no conflict of interest. 


\section{References}

1. Rabinowitz YS. Keratoconus. Surv Ophthalmol 1998; 42: 297-319.

2. Li X, Rabinowitz YS, Rasheed K, Yang H. Longitudinal study of the normal eyes in unilateral keratoconus patients. Ophthalmology 2004; 111: 440-446.

3. Wagner H, Barr JT, Zadnik K. Collaborative longitudinal evaluation of keratoconus (CLEK) Study: methods and findings to date. Cont Lens Anterior Eye 2007; 30: 223-232.

4. Nowak DM, Gajecka M. The genetics of keratoconus. Middle East Afr J Ophthalmol 2011; 18: 2-6.

5. Sahebjada S, Fenwick EK, Xie J, et al. Impact of keratoconus in the better eye and the worse eye on vision-related quality of life. Invest Ophthalmol Vis Sci 2014; 55: 412-416.

6. Pflugfelder SC, Liu Z, Feuer W, Verm A. Corneal thickness indices discriminate between keratoconus and contact lens-induced corneal thinning. Ophthalmology 2002; 109: 2336-2341.

7. Piñero DP, Alió JL, Alesón A, et al. Corneal volume, pachymetry, and correlation of anterior and posterior corneal shape in subclinical and different stages of clinical keratoconus. J Cataract Refract Surg 2010; 36: 814-825.

8. Belin MW, Duncan JK. Keratoconus: The ABCD Grading System. Keratokonus: Das ABCD-System zur Stadieneinteilung. Klin Monbl Augenheilkd 2016; 233: 701-707.

9. Krachmer JH, Feder RS, Belin MW. Keratoconus and related noninflammatory corneal thinning disorders. Surv Ophthalmol 1984; 28: 293-322.

10. Wollensak G, Spoerl E, Wilsch M, Seiler T. Endothelial cell damage after riboflavin-ultraviolet-A treatment in the rabbit. J Cataract Refract Surg 2003; 29: 1786-1790.

11. Hafezi F, Kanellopoulos J, Wiltfang R, Seiler T. Corneal collagen crosslinking with riboflavin and ultraviolet A to treat induced keratectasia after laser in situ keratomileusis. J Cataract Refract Surg 2007; 33: 2035-2040.

12. Wollensak G, Spörl E, Reber F, et al. Corneal endothelial cytotoxicity of riboflavin/UVA treatment in vitro. Ophthalmic Res 2003; 35: 324-328.

13. Flanagan GW, Binder PS. Precision of flap measurements for laser in situ keratomileusis in 4428 eyes. J Refract Surg 2003; 19: 113123.

14. Jonsson M, Behndig A. Pachymetric evaluation prior to laser in situ keratomileusis. J Cataract Refract Surg 2005; 31: 701-706.

15. Gordon MO, Beiser JA, Brandt JD, et al. The Ocular Hypertension Treatment Study; baseline factors that predict the onset of primary open-angle glaucoma; the Ocular Hypertension Treatment Study Group. Arch Ophthalmol 2002; 120: 714-720.

16. Ambrósio R Jr, Klyce SD, Wilson SE. Corneal topographic and pachymetric screening of keratorefractive patients. J Refract Surg 2003; 19: $24-29$.

17. Verdier DD, Sugar A, Baratz K, et al. Corneal thickness as a predictor of corneal transplant outcome. Cornea 2013; 32: 729-736.

18. Grewal DS, Brar GS, Grewal SP. Assessment of central corneal thickness in normal, keratoconus, and post-laser in situ keratomileusis eyes using Scheimpflug imaging, spectral domain optical coherence tomography, and ultrasound pachymetry. J Cataract Refract Surg 2010; 36: 954-964.

19. Konstantopoulos A, Hossain $P$, Anderson DF. Recent advances in ophthalmic anterior segment imaging: a new era for ophthalmic diagnosis? Br J Ophthalmol 2007; 91: 551-557.

20. Izatt JA, Hee MR, Swanson EA, et al. Micrometer-Scale Resolution Imaging of the Anterior Eye In Vivo With Optical Coherence Tomography. Arch Ophthalmol 1994; 112: 1584-1589.

21. Baghdasaryan E, Huang X, Marion KM, et al. Reproducibility of Central Corneal Thickness Measurements in Normal Eyes Using the Zeiss Cirrus 5000 HD-OCT and Pentacam HR. Open Ophthalmol J 2018; 12: 72-83.

22. Ambrósio R Jr, Caiado AL, Guerra FP, et al. Novel pachymetric parameters based on corneal tomography for diagnosing keratoconus. J Refract Surg 2011; 27: 753-758.

23. Hashemi H, Beiranvand A, Yekta A, et al. Pentacam top indices for diagnosing subclinical and definite keratoconus. J Curr Ophthalmol 2016; 28: 21-26.

24. Fujimoto H, Maeda N, Shintani A, et al. Quantitative Evaluation of the Natural Progression of Keratoconus Using Three-Dimensional Optical Coherence Tomography. Invest Ophthalmol Vis Sci 2016; 57: 169-175.

25. Li Y, Meisler DM, Tang M, et al. Keratoconus diagnosis with optical coherence tomography pachymetry mapping. Ophthalmology 2008; 115: 2159-2166.

26. O'Donnell C, Maldonado-Codina C. Agreement and repeatability of central thickness measurement in normal corneas using ultrasound pachymetry and the OCULUS Pentacam. Cornea 2005; 24: 920-924.

27. de Sanctis U, Missolungi A, Mutani B, et al. Reproducibility and repeatability of central corneal thickness measurement in keratoconus using the rotating Scheimpflug camera and ultrasound pachymetry. Am J Ophthalmol 2007; 144: 712-718.

28. Shetty R, Arora V, Jayadev C, et al. Repeatability and agreement of three Scheimpflug-based imaging systems for measuring anterior segment parameters in keratoconus. Invest Ophthalmol Vis Sci 2014; 55: 5263-5268.

29. LiY, Chamberlain W, Tan 0, et al. Subclinical keratoconus detection by pattern analysis of corneal and epithelial thickness maps with optical coherence tomography. J Cataract Refract Surg 2016; 42: 284-295.

30. Mohammadian M, Hashemi H, Jafarzadehpur E, et al. Can OCT pachymetry identify keratoconus suspects. Iranian Journal of Ophthalmology 2014; 26: 189-192.

31. Wang C, Xia X, Tian B, Zhou S. Comparison of Fourier-Domain and Time-Domain Optical Coherence Tomography in the Measurement of Thinnest Corneal Thickness in Keratoconus. J Ophthalmol 2015; 2015: 402925.

32. Kanellopoulos AJ, Asimellis G. Comparison of high-resolution Scheimpflug and high-frequency ultrasound biomicroscopy to anterior-segment OCT corneal thickness measurements. Clin Ophthalmol 2013; 7: 2239-2247.

33. Kiraly L, Stange J, Kunert KS, Sel S. Repeatability and Agreement of Central Corneal Thickness and Keratometry Measurements between Four Different Devices. J Ophthalmol 2017; 2017: 6181405.

34. Prospero Ponce CM, Rocha KM, Smith SD, Krueger RR. Central and peripheral corneal thickness measured with optical coherence tomography, Scheimpflug imaging, and ultrasound pachymetry in normal, keratoconus-suspect, and post-laser in situ keratomileusis eyes. J Cataract Refract Surg 2009; 35: 1055-1062.

35. Yazıc AT, Pekel G, Bozkurt E, et al. Measurements of anterior segment parameters using three different non-contact optical devices in keratoconus patients. Int J Ophthalmol 2013; 6: 521-525. 
36. Nakagawa T, Maeda N, Higashiura R, et al. Corneal topographic analysis in patients with keratoconus using 3-dimensional anterior segment optical coherence tomography. J Cataract Refract Surg 2011; 37: 1871-1878.

37. Kumar M, Shetty R, Jayadev C, Dutta D. Comparability and repeatability of pachymetry in keratoconus using four noncontact techniques. Indian J Ophthalmol 2015; 63: 722-727.

38. Shildkrot Y, Liebmann JM, Fabijanczyk B, et al. Central corneal thickness measurement in clinical practice. J Glaucoma 2005; 14: 331-336.

39. Wickham L, Edmunds B, Murdoch IE. Central corneal thickness: will one measurement suffice? Ophthalmology 2005; 112: 225-228.

40. Berti TB, Ghanem VC, Ghanem RC, Binder PS. Moderate keratoconus with thick corneas. J Refract Surg 2013; 29: 430-435.

41. Ahmadi Hosseini SM, Abolbashari F, Niyazmand H, Sedaghat MR. Efficacy of corneal tomography parameters and biomechanical characteristic in keratoconus detection. Cont Lens Anterior Eye 2014; 37: 26-30.

42. Bae GH, Kim JR, Kim CH, et al. Corneal topographic and tomographic analysis of fellow eyes in unilateral keratoconus patients using Pentacam. Am J Ophthalmol 2014; 157: 103-109 e1.

43. Kamiya K, Ishii R, Shimizu K, Igarashi A. Evaluation of corneal elevation, pachymetry and keratometry in keratoconic eyes with respect to the stage of Amsler-Krumeich classification. Br J Ophthalmol 2014; 98: 459-463.

44. Orucoglu F, Toker E. Comparative analysis of anterior segment parameters in normal and keratoconus eyes generated by scheimpflug tomography. J Ophthalmol 2015; 2015: 925414.

45. Fujimoto K, Inomata T, Okumura Y, et al. Comparison of corneal thickness in patients with dry eye disease using the Pentacam rotating Scheimplug camera and anterior segment optical coherence tomography. PLoS One 2020; 15: e0228567.

46. Barkana Y, Gerber Y, Elbaz U, et al. Central corneal thickness measurement with the Pentacam Scheimpflug system, optical low-coherence reflectometry pachymeter, and ultrasound pachymetry. J Cataract Refract Surg 2005; 31: 1729-1735.

47. Vinciguerra P, Camesasca FI. Prevention of corneal ectasia in laser in situ keratomileusis. J Refract Surg 2001; 17: S187-S189.

48. Zhu X, Zhou Y, Zhang J. Diagnostic value of the pachymetry parameters measured by RTVueOCT for keratoconus. Biomedical Research-tokyo 2017; 28: 5136-5141.

49. Silverman RH, Urs R, RoyChoudhury A, et al. Combined tomography and epithelial thickness mapping for diagnosis of keratoconus. Eur J Ophthalmol 2017; 27: 129-134.

50. Rüfer F, Sander S, Klettner A, et al. Characterization of the thinnest point of the cornea compared with the central corneal thickness in normal subjects. Cornea 2009; 28: 177-180.

51. Avitabile T, Marano F, Uva MG, Reibaldi A. Evaluation of central and peripheral corneal thickness with ultrasound biomicroscopy in normal and keratoconic eyes. Cornea 1997; 16: 639-644.

52. Gherghel D, Hosking SL, Mantry S, et al. Corneal pachymetry in normal and keratoconic eyes: Orbscan II versus ultrasound. J Cataract Refract Surg 2004; 30: 1272-1277. 